\title{
Gobernanza de la administración corporativa en las empresas
} estatales*

\section{Governance of corporate administration in state companies}

\begin{abstract}
Natalia Escobar Escobar **
Melissa Ríos SaRMIENTo** *

* Resultado de investigación del proyecto titulado: Gobierno Corporativo fortalece la gobernanza en las Empresas de Propiedad Estatal. Entidad financiadora: Universidad Libre de Pereira

** Abogada, Especialista en Derecho Empresarial, Magíster en Educación, Doctoranda en Política y Gobierno, docente investigadora asociada de la Universidad Libre-Pereira e integrante del grupo de investigación "Derecho, Estado y Sociedad" (A), adscrito al Centro de Investigaciones Socio-Jurídicas y a la línea de investigación Pensamiento Político y Gestión Pública. correo: natalia.escobare@ unilibre.edu.co ORCID: https://orcid.org// 0000-0002-0268-9441

*** Politóloga, Magister en Derecho Público, investigadora junior reconocida ante Colciencias, docente asociada de la Universidad Libre-Pereira e integrante del grupo de investigación "Derecho, Estado y Sociedad" (A), adscrito al Centro de Investigaciones Socio-Jurídicas y a la línea de investigación DDHH, paz y conflicto. Coordinadora de la regional Risaralda MOE. Correo: melissa.rioss@ unilibre.edu.co ORCID: https://orcid.org/0000-0003-4709-1811
\end{abstract}

Fecha de recepción: Septiembre de 2020

Fecha de aprobación: diciembre de 2020

Para citar este artículo / To reference this article

Escobar, N., Ríos, M. (2020) Gobernanza de la administración corporativa en las empresas estatales. Inciso, 22(2) ;

$247-262$

DOI: http://dx.doi.org/10.18634/incj.22v.2i.1087

\section{Resumen}

Este artículo aborda desde un enfoque sociojurídico la problemática de las empresas de propiedad estatal en la prestación de los servicios públicos que se comprometen a garantizar, resaltando la necesidad de implementar un conjunto de prácticas, procesos y sistemas para dirigir y controlar las empresas de propiedad estatal, de forma tal, que regulen las relaciones entre accionistas y/o propietarios, junta directiva, alta gerencia, y entre la empresa y los grupos 
de interés, para así poder enfrentar los desafíos que se presentan durante la gestión pública, para lo cual se propone el fortalecimiento de la gobernanza, por medio de lineamientos de gobierno corporativo, que lleven a generar dinámicas organizadas, administradas con liderazgo y en ejercicio de la autoridad, mediante procesos de toma de decisiones independientes y deliberadas, con el fin de hacer palpable y eficiente para la prestación del servicio y su impacto en la sociedad. Se evidencia que las Empresas Públicas Estatales-EPE, con frecuencia no generan sistemas, ni procesos eficientes, para dirigir y controlar el desempeño de la misma, y evitar que la toma de decisiones dentro de las juntas directivas este viciada, por lo cual es necesario establecer códigos de buen gobierno corporativo, que fijen normas y estándares de actuación a las juntas directivas, que propendan por una toma de decisiones correcta e independiente, de manera técnica e informada, para que el direccionamiento y liderazgo de la EPE, se base en objetivos claros que alcance metas a corto, mediano y largo plazo.

Palabras clave: Empresas de propiedad estatal, gobernanza, gobierno corporativo.

\begin{abstract}
This article approaches, from socio-legal perspective, the problems of state-owned companies in the provision of public services that they undertake to guarantee, highlighting the need to implement a set of practices, processes and systems to direct and control property-owned companies, so that they regulate relation between shareholders, owners, the board of directors, senior management, and between the company and the interest groups, in order to face the challenges thar arise during public administration for which the strengthening of governance is proposed, through corporate governance guidelines, which lead to the generation of organized dynamics, administered with leadership and in the exercise of authority, through independent and deliberate decision-making processes, in order to make possible and efficient for the provision of service and its impact on society. It is evident that the State Public Companies-SPC, frequently do not generate systems or efficient processes, to direct and control the performance of the same, and avoid that the decision-making within the boards of directors is flawed, for which it is necessary establish Codes of Good Corporate Governance, which establish norms and standards of action for the boards of directors, which promote correct and independent decision-making, in a technical and informed manner, so that the direction and leadership of the SPC is based on objectives clear goals that achieve short, medium and long term.
\end{abstract}

Key words: corporate governance, governance, state-owned companies. 


\section{Introducción}

Las Empresas de Propiedad Estatal (EPE) tienen una gran participación en la provisión de bienes y servicios, tales como, infraestructura, energía, transporte, acueducto, alcantarillado y telecomunicación, entre otros. Por tanto, su funcionamiento y desempeño impactan a toda la población, debido a que son una parte fundamental para el desarrollo económico y social de un país. De esta forma, dichas empresas están llamadas a tener una estructura de administración adecuada que les permita generar estrategias de corto, mediano y largo plazo para poder cumplir así el objetivo para el cual fueron creadas.

Ahora bien, es el Estado quien en este tipo de entes jurídicos (EPE), investido de su condición de propietario actúa dentro de la economía, en forma de empresa, y en la que su participación le otorga control de la misma, por lo que, enfrenta algunos desafíos en aspectos relacionados especialmente con la forma de organización de la propiedad, el relacionamiento con el gobierno, la actuación de la junta directiva, el desempeño de la alta gerencia, los procesos de toma de decisiones (Banco de Desarrollo de America Latina., 2008) y una influencia política indebida por parte del Estado, aspectos que afectan directamente su gobernanza corporativa.

Así entonces, en el caso particular de las EPE es posible afirmar que con frecuencia no generan sistemas ni procesos para dirigir y controlar la empresa de forma adecuada, por lo que la toma de decisiones se ve influenciada por actores políticos de turno, tal como lo afirma la CAF, las juntas directivas: "Están más propensas a privilegiar el interés del Estado y a estar poco orientadas a la visión estratégica y competitiva de la empresa" (Banco de Desarrollo de America Latina, 2017, pág. 17) generando así que se tomen decisiones bajo juicios subjetivos y dependientes de la posición política de turno.

Surge la necesidad de implementar un conjunto de prácticas, procesos y sistemas para dirigir y controlar la empresa, de forma tal que regulen las relaciones entre accionistas y/o propietario, junta directiva, alta gerencia, y entre la empresa y los grupos de interés, para así poder enfrentar los desafíos expuestos anteriormente y fortalecer su gobernanza corporativa. Esto es precisamente, por lo que propende el Gobierno corporativo. En consecuencia, es factible plantear la siguiente pregunta: ¿Cómo la implementación del gobierno corporativo fortalece la gobernanza en las empresas de propiedad estatal?

Así entonces, en el presente documento se abordarán temáticas conducentes a absolver este interrogante como son: i) las generalidades de los conceptos correspondientes a categorías jurídicas estrechamente relacionadas con el tema de gobierno corporativo y sus problemáticas, ii) los desafíos a los que se enfrenta la gobernanza en las Empresas de Propiedad Estatal, así como iii) los lineamientos del Gobierno Corporativo que fortalecen la gobernanza.

Para desarrollar estos objetivos, se recurrió al análisis de la información recolectada, de tipo bibliográfica, jurídica, investigaciones académicas e informes de organismos internacionales y nacionales, para comprender el fenómeno abordado y sus dinámicas, y a partir de esto, generar relaciones y recomendaciones sobre maneras más eficientes del uso de dineros públicos por medio de la gestión corporativa en empresas de carácter estatal. A raíz de lo anterior, esta es una investigación de tipo sociojurídico, con un enfoque cualitativo y analítico. 


\section{Gobierno corporativo}

El gobierno corporativo es un conjunto de prácticas, procesos y sistemas para dirigir y controlar una empresa, de esta forma, es un instrumento o herramienta que puede contribuir a regular las relaciones entre accionistas y/o propietario, junta directiva, alta gerencia y entre la empresa con los grupos de interés, fortaleciendo así la disposición ética de la compañía (Banco Interamericano de Desarrollo y Bolsa de Valores de Colombia, 2009).

La Corporación Financiera Internacional (CFI), ha señalado que la expresión gobierno corporativo hace referencia a las estructuras y procesos específicamente diseñados para la dirección y el control de las compañías, ocupándose de las relaciones entre alta gerencia, junta directiva, accionistas controladores, accionistas minoritarios y/o propietarios y otras partes interesadas; contribuyendo al desarrollo económico sostenible y también a mejorar el desempeño de las compañías e incrementar su acceso al capital externo. (Cfr: International Finance Corporation, 2019).

Para Rodríguez Jaraba, la noción de gobierno corporativo es una declaración de principios de autorregulación, plasmada en lineamientos prácticos de conducta y desempeño establecidos en procesos, procedimientos, hábitos y costumbres preferidos por la administración de las empresas. También es considerado como un compendio de principios, valores y convicciones expresados a manera de postulados, orientados a maximizar la gestión administrativa de las empresas, y con ello, a trasmitir confianza, prevenir problemas y mitigar riesgos (Rodriguez, 2014).

Además de lo anterior, es posible afirmar que el gobierno corporativo privilegia la ética, la legalidad, la transparencia, la objetividad y la competitividad sobre las prácticas administrativas. Para García Soto, por consiguiente, cualquier manifestación del gobierno corporativo se debe fundamentar principalmente en los principios de buena fe, justicia y equidad, y sus mandamientos se deben soportar en la eficiencia, la eficacia y la economía de los procesos que siguen las organizaciones en ejercicio de sus actividades productivas (Garcia , 2003).

Consecuente con lo expresado, la Organización para la Cooperación y el Desarrollo Económico (OCDE,2017), determina que el término gobierno corporativo hace referencia al sistema a través del cual los negocios son dirigidos y controlados, especificando la distribución de derechos y responsabilidades entre los diferentes participantes de la corporación, tal como el consejo, dirección, accionistas y otros grupos de poder; definiendo las reglas y procedimientos para la toma de decisiones en los temas corporativos; y proporcionando la estructura a través de la cual los objetivos de la compañía son fijados, así como los medios para lograr esos objetivos y controlar la rentabilidad.

Según el profesor Julián Benavides Franco: “El tema de gobierno corporativo se origina con la idea de la empresa moderna. Se identifica inicialmente con la delegación del poder sobre la toma de decisiones hacia administradores distintos a los dueños," (Benavides, 2003, págs. 1-10) Así, el término gobierno corporativo ha evolucionado de un concepto financiero, relativo al retorno sobre la inversión, exigido por los inversionistas, a uno que incluye aspectos relativos al diseño de la organización misma que tiene que ver con los medios internos por los cuales las corporaciones son operadas y controladas. 
De otro lado, el Banco de Desarrollo de América Latina - CAF, ha desarrollado el triángulo de gobierno corporativo que representa el modelo de gobernabilidad en el que ha establecido un balance entre tres grupos que forman el triángulo:

- Propietarios e inversionistas que aportan capital para financiar el negocio.

- Los ejecutivos, gerentes y empleados que manejan el negocio en el día a día e implementan las políticas y estrategias.

- Juntas Directivas, que mantienen cuatro funciones principales, (i) representar los intereses de los propietarios, (ii) supervisar y brindar asesoramiento estratégico a la dirección ejecutiva (iii) establecer políticas que apoyen el objeto social; y (iv) cumplir con su deber legal de actuar en el mejor interés de la empresa. (Bernal, Penfold, Lisa, \& Wilcox, 2012, pág. 29).

De esta manera se muestra que, al proporcionar condiciones óptimas reflejadas en el triángulo, cada parte puede cumplir con sus responsabilidades y desarrollar sus funciones, alcanzando así los objetivos estratégicos para lograr un desarrollo sostenible a largo plazo. En resumen, para conservar el equilibrio dentro del triángulo de gobierno corporativo, es indispensable que cada uno de los actores conozca sus responsabilidades. Por ejemplo, la junta directiva debe aportar a la propiedad mecanismos con los que pueda conocer la actuación individual de sus miembros, mediante sistemas de evaluación de desempeño. A su vez, la propiedad debe aportar a la junta directiva un adecuado nombramiento, remoción y ratificación de miembros de sus miembros, por su parte, la junta directiva debe aportar a la dirección conocimientos, contactos y seguimiento al desempeño de la dirección. A su vez, la dirección tomará decisiones sobre los recursos que se van a invertir, por lo cual deberá presentar informes de rendición de cuentas, siguiendo buenas prácticas. (Gomez, 2020).

Ahora bien, teniendo en cuenta que el presente documento se desarrollará en el marco del gobierno corporativo en las Empresas de Propiedad Estatal a nivel nacional, a continuación, se realiza un acercamiento al concepto Empresa de Propiedad Estatal en Colombia - EPE.

\section{Gobernanza corporativa}

El concepto de gobernanza se ha estudiado desde diferentes puntos de vista y a su vez ha sido entendido de múltiples maneras, pero para efectos del presente trabajo se tomará como referencia el concepto de gobernanza corporativa. Según Francisco Ganga y Osma Núñez, definen el concepto de gobernanza: "Como el estudio de todos los mecanismos, procesos y reglas a través de los cuales se ejerce la autoridad económica, política y administrativa de una organización" (Ganga \& Nuñez, 2018, pág. 9).

A su vez, Luis F. Aguilar, plantea que la gobernanza representa un:

Proceso de dirección, con participación de varios actores en el diseño y ejecución de sus objetivos; que requiere de una estructura en tanto define objetivos orientados a la acción social y normas causales de producción, que proceden de los resultados probados de ciencia, tecnología y gerencia. (Aguilar, El Concepto de Gobernanza., 2006, pág. 11) 
A partir de estos resultados, el Estado prestará y distribuirá bienes y servicios públicos entre sus conciudadanos, lo cual se complementa con lo escrito por, el rector e investigador de la Universidad de Lasalle de Canoas- Brasil, Paulo Fossatti, quien definió que la: "Gobernanza comprende la gestión de una organización que involucra procesos y reglas, que garantizan los valores, el bienestar y el avance en el conocimiento y la calidad de una organización" (Ganga \& Nuñez, 2018, pág. 10).

No obstante, lo anterior, es importante hacer una diferenciación entre los conceptos gobernanza y gobernabilidad, están ligados, pero son diferentes. Por lo tanto, cuando se habla de gobernabilidad, se habla de la capacidad para dirigir-liderar, depende de las decisiones que se tomen para responder a las necesidades sociales (Camou, 2001), y en cuanto a gobernanza, se refiere a la armonía entre todos los factores, normas, procesos y actores, involucrados en la prestación del servicio público que responderá a las necesidades iniciales, pues de esta depende su éxito y real impacto.

De acuerdo con lo expuesto, se tomará el concepto de gobernanza corporativa como la forma en que son organizadas, estructuradas y controladas las organizaciones, esto unido al modo de administrarlas con liderazgo y mediante el proceso de toma de decisiones, para el ejercicio de la autoridad, legitimada por las acciones y la construcción de acuerdos.

Finalmente, dentro del presente artículo se expusieron los conceptos de gobierno corporativo, de Empresas de Propiedad Estatal y de gobernanza corporativa, para el entendimiento conceptual y así poder responder la pregunta formulada en la introducción. De este modo, se da paso al siguiente acápite, donde se abordarán los desafíos de la gobernanza en las EPE, teniendo en cuenta que, el universo de las empresas estatales presenta particulares desafíos en especial los que se relacionan con la gobernanza, como lo son las formas de organización, la función de propiedad y el relacionamiento de los órganos de administración, estos comprendidos en el triángulo de gobierno corporativo.

\section{Desafíos de la gobernanza en empresas de propiedad estatal-EPE}

En la actualidad las EPE tienen una gran participación en la provisión de bienes y servicios, tales como, infraestructura, energía, transporte, acueducto, alcantarillado y telecomunicaciones. En este sentido, podría afirmarse que su funcionamiento y desempeño impacta a toda la población, pues es parte fundamental del desarrollo económico y social. En palabras de (Hierro \& Herrera, 2019), las EPE son un:

Instrumento de intervención del poder público en la economía, mediante la producción de bienes y servicios, en cualquier sector de la actividad, organizado en forma de empresa, que en principio se financia con las contraprestaciones recibidas de sus clientes y en la que la participación del Estado como propietario le otorga control de esta. (pág. 52)

Así entonces, las EPE enfrentan algunos desafíos en aspectos relacionados especialmente con la forma de organización de la propiedad, el relacionamiento con el gobierno, la actuación de la junta directiva, el desempeño de la alta gerencia, los procesos de toma de decisiones y la influencia política indebida por parte del Estado (Fontes, 2008), aspectos que afectan 
directamente la gobernanza corporativa; debido a lo anterior es que en ocasiones se cuestiona su idoneidad para efectivamente cumplir los objetivos para los que fueron creados (Banco de Desarrollo de America Latina CAF, 2017).

La gobernanza en las EPE enfrenta diferentes desafíos relativamente mayores que en las empresas privadas, pues, estas empresas tienen en general objetivos múltiples que contrastan con el objetivo de maximización de valor para las empresas privadas. Dichos objetivos pueden llegar a ser contradictorios cuando distintas partes interesadas entran en conflicto y, en cualquier caso, generan dificultades para el desarrollo y cumplimiento de sus objetivos empresariales (Vives, 2007).

A manera de ejemplo, encontramos que las EPE que tienen como objeto social la prestación de servicios públicos, enfrentan a nivel de su gobernanza decisiones estratégicas que oscilan entre las directrices del mercado y la materialización de los fines sociales del Estado, pues, este se mueve en diferentes roles como propietario o accionista de una empresa y como garante de la finalidad social del Estado, tal como lo expone el artículo 365 de la Constitución Política.

Es por lo anterior que, en desarrollo del presente acápite, se realizará una aproximación a la gestión del estado propietario, a los desafíos de la gobernanza en las EPE y a la injerencia política a la cual se ven expuestas las EPE.

\section{El Estado propietario}

Según el CONPES 3851 de 2015 (Consejo Nacional de Política Económica y Social. Departamento de Planeación Nacional., 2015), el Estado tiene unas características particulares como propietario de empresas, esto le permite participar dentro de sectores estratégicos para el desarrollo económico del país, resolver fallas o disposiciones del mercado o del gobierno y tener participación accionaria de alta rentabilidad como fuente de ingresos fiscales. De este modo, el Estado comienza a asumir diferentes roles, no solo como regulador, diseñador de políticas públicas o garante de la sostenibilidad fiscal, también debe velar por su carácter de propietario y/o accionistas, generando así que entre en conflicto con algunas de las funciones estatales, ya que el Estado no es un accionista ordinario como lo prevé el Código de Comercio, por lo tanto, no se presume el ánimo de lucro. Sin embargo, una vez opta por ser propietario, debe acogerse a las consecuencias jurídicas, esto conlleva a que cambie en su actividad y su naturaleza, comportándose como un propietario donde sus empresas deben generar rentabilidad.

Así entonces, el Estado se enfrenta al reto de encontrar un equilibrio entre su responsabilidad de ejercer efectivamente sus funciones de propiedad y abstenerse de realizar interferencias políticas de corto plazo que comprometen la sostenibilidad de sus empresas a largo plazo, pues su rol de Estado de servir al interés púbico, y las prácticas políticas que desarrollan sus actores, interfiere en el cumplimiento de los propósitos empresariales.

Por otra parte, según Germán Coloma, las EPE desde el inicio tienen problemas de agencia, puesto que el verdadero propietario de los activos que es el Estado (principal), que delega su representación en los órganos de tipo político (agente), que a su vez encomienda su administración a una estructura burocrática; dado que los primeros delegan en los segundos 
la administración de la empresa, el principal (Estado) desconoce con exactitud el estado de los negocios administrados, perdiendo así su capacidad para dirigir la empresa, pues no puede coordinar sus actores y normas; generando así, la pérdida de su gobernanza y gobernabilidad (Coloma, s.f.).

Para Sanclemente, esto es, los riesgos generados en el problema de agencia, se incrementan debido a que el nivel de delegación se profundiza. En efecto, el principal es el Estado, que en ultimas es el propietario, y delega en sus responsables políticos la dirección de sus empresas, esto se refleja en la legislación colombiana en la Ley 489 de 1998, en donde el presidente, gobernador $\mathrm{o}$ alcalde tienen la potestad de nombrar gerentes y juntas directivas de sus empresas a cargo, de esta manera, se aprecia lo dicho, pues la distancia entre el principal y el agente es mayor, intensificando los riesgos de conflictos de interés, en la medida en que cada una de las instancias de decisión mencionadas, se puede ver tentada a imponer su propio provecho a los intereses del principal, es por esto que, es importante garantizar el control efectivo de la gestión empresarial (Sanclemente, 2019).

A su vez, Alexander Kanov, considera que el Estado cuando está en el papel de empresario propietario resulta ser ineficaz, según la ideología política neoliberal del mercado, el Estado solo debe intervenir como regulador en el mercado, mas no como propietario, porque entre las: "Causas del bajo rendimiento de la propiedad estatal algunos investigadores y funcionarios gubernamentales mencionan su calidad de no pertenecer a nadie, pues la propiedad estatal es la propiedad no personificada" (Kanov , 2003), es la propiedad de todos y de nadie, por lo cual, es importante que el Estado defina políticas de propiedad, de otro modo, las empresas tendrían un comportamiento deficitario.

Ahora bien, otro de los problemas o desafíos de la gobernanza que surgen dentro de las EPE, está en su estructura corporativa, en donde se deben tomar las decisiones estratégicas, lo cual se tratará en el siguiente acápite.

\section{Aspectos de la estructura organizacional}

La estructura organizacional desempeña tres funciones básicas: La primera, producir resultados y alcanzar objetivos organizacionales; la segunda, para regular o minimizar la influencia de las diferencias individuales sobre la organización; la tercera, tomar decisiones sobre el funcionamiento de la organización (Morejon \& Maryury, 2016).

Para el análisis de este acápite, se hará énfasis en la tercera función, de la cual hacen parte los órganos de dirección y de control, que son: propietarios, ejecutivos, gerentes, alta gerencia y la junta directiva, como se evidenció en la primera parte de este artículo, por medio del triángulo del gobierno corporativo. Desde la visión del Estado propietario y/o accionista, se tomará a la junta directiva como el centro de atención para el desarrollo de esta parte.

Al respecto, la OCDE se ha pronunciado afirmando que, una de las principales deficiencias que generan fallas en la estructura corporativa, está en la conformación de las juntas directivas y como estas toman sus decisiones, tal como lo expresa (Sanclemente , 2019), el pobre desempeño 
de la propiedad accionaria estatal estaría relacionada con la falta de experiencia, ya que el Estado no sabe ser propietario, y una de las razones es porque los responsables de la toma de decisiones están influenciados por políticos, esto hace que las decisiones estratégicas estén permeadas de posiciones e intereses del gobierno de turno, ocasionando en algunos momentos, que sean decisiones antitécnicas y poco estratégicas.

La importancia de los criterios técnicos se refleja en la independencia de criterios que deben tener los administradores cuando toman una decisión. Se consideran administradores los referenciados en el artículo 22 de la Ley 222 de 19951. Por lo tanto, respecto a la toma de decisiones estratégicas, no solo hacen parte de ello, la junta directiva o los consejos de administración (artículo 22 de la Ley 1995), sino también los altos directivos, de allí la necesidad de personas idóneas y líderes.

El proceso de toma de decisiones empresariales como un proceso grupal, un esfuerzo planeado y cooperativo, en el cual cada participante tiene un papel reconocido que cumplir y unas obligaciones o tareas por ejecutar, que se le asignan para cumplir los objetivos de la organización más que para satisfacer preferencias individuales. (Cabeza de Vergara, Muñoz, \& Vivero, 2004, pág. 130)

\section{Injerencia política}

Según el Banco de Desarrollo de America Latina (2017), la influencia política indebida por parte del Estado afecta el objetivo sobre el cual fueron creadas las EPE y su sostenibilidad a largo plazo, puesto que los intereses políticos del momento, impiden cumplir sus obligaciones en pro del objeto general de la empresa, ya sea este social o económico, tal como lo plantea la (OCDE, 2011), al exponer que las EPE pueden sufrir tanto por un intervencionismo indebido por parte del Estado como propietario o controlante debido a motivaciones políticas como por un Estado propietario totalmente pasivo.

Estos comportamientos interesados de políticos o burócratas, mal llamado a veces injerencia política (sin desconocer que esta puede tener un aspecto positivo), ha sido un factor latente en el ámbito nacional, para el legislador colombiano, de allí la necesidad de salvaguardar a las EPE de dichos comportamientos, por ejemplo, en el sector de servicios públicos domiciliarios, la Ley 142 de 1994 en su artículo 27.3 define que, la Nación, las entidades territoriales, y las entidades descentralizadas:

Deberán exigir a las empresas de servicios públicos, una administración profesional, ajena a intereses partidistas, que tenga en cuenta las necesidades de desarrollo del servicio en el mediano y largo plazo. Al mismo tiempo es derecho suyo fijar los criterios de administración y de eficiencia específicos que deben buscar en tales empresas las personas que representen sus derechos en ellas, en concordancia con los criterios generales que fijen las comisiones de regulación.

Por lo que se puede apreciar que desde la normatividad ya se vislumbran señales regulatorias que propenden porque la administración de las empresas de servicios públicos debe ser ajena a intereses partidistas, lo cual en la realidad no ocurre. Dentro del mismo articulado de la Ley 142

1. Art. 22 Ley 222 de 1995: "Son administradores, el representante legal, el liquidador, el factor, los miembros de juntas o consejos directivos y quienes de acuerdo con los estatutos ejerzan o detenten esas funciones". 
de 1994, el nominador de la junta directiva y del gerente general es el presidente, gobernador o alcalde, lo que genera que los nombramientos sean de forma discrecional, pues son mal denominados "cargos de confianza" lo que genera perfiles poco profesionales que les impide cumplir a cabalidad las responsabilidades del cargo. Además, esto incentiva a que el grupo de miembros que conforma los órganos de administración y formuladores de las estrategias, estén mediados por intereses partidistas, contrario al deber y exigencia planteado en el artículo 27.3 de la Ley 142 de 1994 anteriormente expuesto a manera de ejemplo.

De lo expuesto, se observa que el problema no es que las empresas sean públicas como tal, sino que existe una injerencia política inadecuada a la cual se ven enfrentadas cada periodo electoral desde su creación hasta la actualidad.

Para concluir se resalta que al Estado propietario le es muy difícil competir en el mercado porque; primero, la propiedad es de todos y a la vez de nadie, esto genera que cuando hay perdidas no exista un real y verdadero afectado directo; segundo, el Estado actúa desde dos roles, como propietario accionista y como garante de la finalidad social del Estado; tercero, el Estado propietario no tiene control sobre las injerencias políticas que se presentan dentro de la toma de decisiones, su órgano principal de dirección que es la junta directiva, se ve afectado por los ciclos políticos que generan cambios continuos de sus integrantes, lo que altera su capacidad para desarrollar planes a largo plazo, imponiendo costos sobre la productividad y la rentabilidad además; cuarto, las juntas directivas normalmente son conformadas por miembros poco profesionales, y no son independientes del gobierno central o el propietario; quinto, la elección de las juntas directivas, su tamaño, la cantidad de miembros, los procesos y mecanismos de lección, el perfil educativo y profesional y la independencia de sus miembros genera que no haya una adecuada estructura para la toma de decisiones estratégicas. (Bernal, Penfold, Lisa, \& Wilcox, 2012).

Ahora bien, la cuestión central no se refiere entonces a si debe o no existir las EPE, sino al tipo de gobernanza y orientación estratégica que deben tener. Por lo tanto, el gobierno corporativo tal como se definió en el primer aparte, es una de las opciones que contribuye a fortalecer la gobernanza de este tipo de entidades, de tal manera que, puedan adoptar decisiones independientes.

Se pasará aborda los mecanismos que el gobierno corporativo ofrece para fortalecer dicha gobernanza.

\section{Lineamientos del gobierno corporativo que fortalecen la gobernanza en las EPE}

Teniendo en cuenta lo expresado anteriormente en donde se expusieron las nociones generales de gobierno corporativo, de empresas de propiedad estatal, de gobernanza corporativa y se presentaron los desafíos a los que se enfrentan las EPE en aspectos relacionados con la gestión de la propiedad, el relacionamiento con el gobierno, la actuación de la junta directiva y los procesos de toma de decisiones que afectan directamente la gobernanza corporativa; se pretende exponer porqué el gobierno corporativo es importante para las EPE, ya que un gobierno corporativo sólido, genera una buena gobernanza.

Así entonces, es posible decir, que el gobierno corporativo en las EPE podría actuar como carta 
de navegación para la administración pública, puesto que, no solo ayuda a monitorear el logro de sus objetivos, sino también a que haya una línea de mando con responsabilidades claras por parte del Estado como propietario, de las juntas directivas como instancia estratégica y de la alta gerencia como ejecutores de dichas estrategias (Nabil \& Oneto, 2015).

La OCDE teniendo en cuenta que las EPE ocupan una posición destacada en sectores de servicios básicos como energía, transporte, acueducto, alcantarillado, infraestructura y telecomunicaciones, en 2011 publicó las Directrices sobre el Gobierno Corporativo de las Empresas Públicas (OCDE, 2011), dirigidas fundamentalmente a empresas comerciales que son propiedad del gobierno central y federal, que además resultan útiles para empresas no comerciales que realizan funciones especialmente de política pública. El documento presenta las siguientes directrices: I) definir un marco jurídico y regulatorio efectivo para las EPE, II) el Estado debe actuar como propietario, III) mantener un tratamiento equitativo entre accionistas, IV) manejar con transparencia las relaciones con partes interesadas, V) tener políticas de transparencia, rendición de cuentas y difusión de la información y VI) la responsabilidad de los directivos de empresas públicas en pensar y asumir la responsabilidad de sus decisiones. De esta forma, se promueve que el sector público busque sistemas de organización que le garanticen ser más eficientes y eficaces en su gestión.

De tal manera se plantea que, teniendo en cuenta las directrices de la OCDE, es posible enfrentar los desafíos expuestos, por lo tanto, se pasará a exponer los lineamientos del gobierno corporativo que permiten gestionar al Estado propietario y contribuyen a mantener la gobernanza corporativa.

\section{Lineamientos de gobierno corporativo que permiten gestionar al Estado propietario}

Las EPE normalmente tienen un doble propósito, obtener ganancias dentro de una práctica empresarial sostenible y servir al interés público, ya que el propietario es el Estado. Así entonces, es necesario encontrar un equilibrio que le permita al Estado efectivamente ejercer sus funciones de propiedad absteniéndose de intervenir políticamente dentro de las decisiones estratégicas, y propendiendo por establecer un marco legal y regulatorio claro para la actuación de las EPE en todos sus aspectos, y esto puede lograrse, por ejemplo, adoptando en la mayor medida posible las directrices corporativas planteadas por la OCDE para empresas públicas, con el fin de favorecer la competencia en igualdad de condiciones en el sector público (Vives, 2007).

A su vez, dentro de las directrices de la OCDE, se menciona que el Estado debe; primero, aclarar y priorizar sus objetivos; segundo, tener claras sus prioridades; tercero, abstenerse de interferir en los temas operativos, respetando la independencia de los órganos tomadores de decisiones, esto es la junta directiva; cuarto, como prioritario, construir una política de propiedad clara, consistente y explícita que proporcione la posibilidad de plantear y cumplir sus objetivos a largo plazo, para que no esté mediado por los periodos políticos a los que normalmente se ven expuestas las EPE. Lo anterior, se refiere a que debe haber una separación clara entre los diferentes papeles que el Estado ejerce, pues su papel de propietario debe estar separado de su papel de regulador del mercado y de garante del cumplimiento de los fines del Estado social de derecho (Vives, 2007). 
De esta forma, el Estado propietario debe respetar la autonomía de las EPE otorgada por la descentralización administrativa, creando así las condiciones para una adecuada gobernabilidad. Por esto, se deberán construir acuerdos voluntarios entre el propietario ya sea nación, departamento o municipio y la EPE, que constituyen convenios marcos de relaciones o convenios de gobernabilidad suscritos a través de sus representantes, mediante los cuales se definan los lineamientos dentro de los que se desarrolle la relación propietario - empresa, los principios sobre los cuales va a regir su relación, las obligaciones y responsabilidades de cada uno, tendientes a mejor el desempeño de la empresa, en términos de rentabilidad y sostenibilidad de forma tal que garantice el buen manejo de sus inversiones. (Alcaldía de Medellín, 2007).

Esto es, en palabras de Sanclemente (2019), que el Estado accionista debe adoptar medidas que se dirijan a garantizar que el poder que ejerce sobre sus empresas se exprese a través de instrumentos jurídicos con que, por ejemplo, con los que cuentan los accionistas en las sociedades anónimas, tal como lo recomienda la OCDE, las EPE deben reconocer los derechos de todos los accionistas minoritarios, brindando un trato equitativo, manteniendo un nivel elevado de transparencia, desarrollando una política adecuada de acceso a la información y facilitando la participación dentro de las juntas generales de accionistas para que puedan ser parte de la toma de decisiones empresariales (OCDE, 2011).

Lo dicho, contribuye a que las EPE puedan tener autonomía y el Estado solo participe dentro de los límites que le permite el acuerdo, de esta manera la EPE no se verá avocada a los cambios políticos de turno, lo que permitirá además que pueda fortalecer su gobernanza.

Respecto al gobierno corporativo, en este punto, es importante mencionar que en el mundo existen dos sistemas uno basado en principios y otro basado en normas. En el caso de las EPE el sistema de gobierno corporativo que más se adopta de manera voluntaria es el basado en la adopción de un Código de Buen Gobierno Corporativo (Bernal, Penfold, Lisa, \& Wilcox, 2012) mediante el cual se fijan normas y estándares de actuación para las juntas directivas, exigiendo transparencia en los procesos decisorios y rendición periódica de cuentas de las decisiones tomadas, también, establece vías formalizadas de acceso de los accionistas, interesados y miembros de la empresa a la información sobre las propuestas de decisión, las decisiones adoptadas, las acciones ejecutadas, los recursos ejercidos y los resultados obtenidos (Aguilar, 2014). Por lo tanto, es claro que el gobierno corporativo se involucra sustancialmente en la gobernanza de las EPE, pues estas no son ajenas a los sistemas de planeación, organización, dirección y control (Diaz, Garcia, \& Garcia, 2018).

\section{Limitación de la injerencia política}

Tal como se mencionó, uno de los desafíos que enfrentan las EPE es la influencia política indebida por parte del Estado (Banco de Desarrollo de America Latina CAF, 2017), que genera presiones debido a sus intereses políticos de turno, afectando así los procesos de toma de decisiones y su desempeño a largo plazo. Así entonces, mediante el gobierno corporativo se puede generar un ambiente sólido y objetivo para el desarrollo empresarial, pues blinda a la empresa de injerencias políticas, tal como lo plantea la OCDE en sus Directrices sobre Gobierno 
Corporativo para las Empresas Públicas, al exponer que el Estado deberá abstenerse de interferir en los temas operativos, e involucrarse en la gestión cotidiana de la empresa, respetando la independencia del directorio (junta directiva). Una política clara ayudará a evitar situaciones en las que las empresas públicas sean obstaculizadas por el Estado pues estas son autónomas; administrativa y financieramente, para fijar sus propios objetivos.

Como se expuso en el acápite de lineamientos de gobierno corporativo para gestionar al Estado propietario, se debe construir una política de propiedad clara, consistente y explicita, lo que proporcionará previsibilidad hacia el mercado y los grupos de interés en general, así como una comprensión clara de los objetivos y labores del Estado como propietario.

Un ejemplo de lo anterior es lo ocurrido con algunas empresas chinas, pues estas experimentaron una reforma de propiedad, a través de la cual fueron cotizadas o privatizadas, lo que generó una pérdida o reducción del poder político (Rojas, 2015). Se podría decir que gracias a la nueva estructura empresarial se logró limitar la injerencia política indebida. Esto no quiere decir que sea el mejor camino para fortalecer la gobernanza de las EPE, pues el Estado, según la OCDE, puede ejercer un propiedad activa e informada, sin involucrarse en la gestión cotidiana de la empresa. Un ejemplo de ello son las Empresas Públicas de Medellín (EPM), que permanece 100\% pública y con un gobierno corporativo sólido, pues delimitó los roles entre el propietario y los órganos de administración (triángulo de gobierno corporativo), impulsó una cultura transparente y ética frente al propietario y los grupos de interés e implementó mecanismos de autorregulación y garantías para mantener la independencia y objetividad en el desarrollo de sus objetivos (Bernal, Penfold, Lisa, \& Wilcox, 2012).

Finalmente, mediante los lineamientos del gobierno corporativo es posible establecer una armonía entre las relaciones del propietario, la alta gerencia y la junta directiva, lo que se hace relevante para mantener una buena gobernanza corporativa, entendida esta, como la forma en que son organizadas, estructuradas y controladas las organizaciones, unido al modo de administrarlas con liderazgo y mediante un proceso de toma de decisiones independientes, en el ejercicio de su autonomía y legitimadas por acciones construidas bajo acuerdos comunes.

\section{Conclusiones}

En las últimas décadas el sector público ha venido buscando nuevos sistemas de organización que le garanticen ser más eficiente y eficaz en su gestión (Cabeza de Vergara, Muñoz, \& Vivero, 2004), pues acarrea diferentes desafíos, en su organización de la propiedad, el relacionamiento con el gobierno, la actuación de la junta directiva, el desempeño de la alta gerencia y los procesos de toma de decisiones, que afectan directamente su gobernanza corporativa, pero que según todo lo expresado en este artículo la implementación del gobierno corporativo la fortalece porque:

Primero, el Estado tiene un doble propósito que es obtener ganancias dentro de una práctica empresarial sostenible y servir al interés público. Siguiendo los lineamientos del gobierno corporativo, el Estado como propietario, podrá gestionarse de tal manera que mediante acuerdos marcos de gobernabilidad, regule su interferencia o pasividad con la empresa de la cual es propietario, por tal motivo, deben quedan claros los lineamientos de gobierno corporativo. 
Segundo, mediante la implementación del gobierno corporativo, es posible llevar a cabo mecanismos transparentes y permanentes en la elección de sus miembros de junta directiva del gerente o presidente, para que estos sean sujetos con altas capacidades profesionales y personales, que permitan que la toma de decisiones sea de forma correcta, de manera técnica, e informada y de modo independiente, para que así sea posible que el direccionamiento se base en objetivos claros y se fijen metas a largo plazo.

Tercero, el triángulo de gobierno corporativo permite que sus actores se relacionen de manera armónica, donde a cada uno se le asignan responsabilidades claras, para poder así mantener un buen sistema de pesos y contrapesos que permita tomar decisiones adecuadas, esto es decisiones técnicas e independientes de los vaivenes políticos a los que se enfrentan este tipo de empresas.

Cuarto, el establecer Códigos de Buen Gobierno Corporativo permite que se fijen normas y estándares de actuación especialmente de la junta directiva donde se exige transparencia en los procesos decisorios, una rendición de cuentas periódicas y vías formalizadas de acceso a la información de actores internos y externo de la empresa.

De este modo, es el gobierno corporativo es el que contribuye a mantener la gobernanza de las EPE para que así se logre establecer unas dinámicas de dirección que se den de forma organizada y bien administrada, con liderazgo y ejercicio de la autoridad, mediante procesos de toma de decisiones independientes, legitimadas por acciones salientes de la construcción de acuerdos.

\section{Referencias bibliográficas}

Aguilar, L. (2006). El Concepto de Gobernanza. Flacso, CRI y FOCAL, Ciudad de Mexico.

Aguilar, L. (2014). Las dimensiones y los niveles de la gobernanza. Cuadernos de Gobierno y Administración Pública. Sistema Nacional de Investigadores.

Alcaldia de Medellin. (2007). Convenio Marco de Relaciones Municipio de Medellín . Medellin: Empresas Publicas de Medellin E.S.P.

Banco de Desarrollo de America Latina. (2017). Efectividad y estructura de los Directorios de las Empresas de Propiedad del Estatal en América Latina y el Caribe. Serie Politicas Publicas y transformacion productiva(26).

Banco de Desarrollo de America Latina. (2017). Efectividad y estructura de los Directorios de las Empresas de Propiedad del Estatal en América Latina y el Caribe. Serie Politicas Publicas y Transformacion Productiva(26).

Banco de Desarrollo de America Latina CAF. (2017). Efectividad y estructura de los Directorios de las Empresas de Propiedad del Estatal en América Latina y el Caribe. Serie de Politicas Publicas y Transformacion Porductiva(26). 
Banco de Desarrollo de America Latina. (2008). Gobernabilidad corporativa en Empresas Estatales y el Papel del Consejo de Administración. Revista Chilena de Administracion Pùblica, 11, 59-87.

Banco Interamericano de Desarrollo y Bolsa de Valores de Colombia. (2009). Desarrollo de Instrumentos Informativos sobre el Mercado de Capitales. Gobierno Corporativo. Bogota: Colombia Capital.

Benavides, F. (2003). Historia del Gobierno Corporativo en el Mundo. Definición y objetivos del concepto de Gobierno Corporativo. En Confecàmaras, Libro Blaco Gobierno Corporativo (págs. 1-10). Colombia: Confecàmaras.

Bernal, A., Penfold, M., Lisa, S., \& Wilcox. (2012). Gobierno Corporativo en América Latina. Importancia para las Empresas de Propiedad Estatal. Serie Politicas Publicas y Transformacion Porductiva(6).

Cabeza de Vergara, L., Muñoz, S., \& Vivero, S. (2004). Aproximación al proceso de toma de decisiones en la empresa barranquillera. Pensamiento y gestion(17), 1-38.

Camou, A. (2001). Gobernabilidad y Democracia. Cuaderno de divulgacion de la cultura democratica (4ta ed., Vol. 6). Ciudad de Mexico: Instituto Federal.

Cfr: International Finance Corporation. (2019). External Corporate. Obtenido de http://www. ifc.org/wps/wcm/ connect/Multilingual_Ext_Content/IFC_External_Corporate_Site/ Home_ES. 04/02/2020

Coloma, G. (s.f.). Empresa pública, privatización, regulación y competencia: su papel en la provisión de servicios de infraestructura. Obtenido de Universidad CEMA: https:// ucema.edu.ar/publicaciones/download/documentos/268.pdf

Consejo Nacional de Política Económica y Social. Departamento de Planeación Nacional. (2015). Política General de Propiedad de Empresas Estatales del Orden Nacional. CONPES 3851.

Diaz, A., Garcia, D., \& Garcia, C. (2018). El Gobierno Corporativo en el sector publico en Colombia: Una Aproximación. Revista Espacios, 39(48).

Fontes, J. (2008). Gobernabilidad corporativa en Empresas Estatales y el Papel del Consejo de Administración. Revista Chilena de Administracion Publica(11), 59-87.

Ganga, F., \& Nuñez, O. (2018). Gobernanza de las Organizaciones: Acercamiento conceptual a las instituciones de Educación Superior. Revista Espacios, 39, 9.

Ganga, F., \& Nuñez, O. (2018). Gobernanza de las Organizaciones: Acercamiento conceptual a las instituciones de Educación Superior. Revista Espacios, 39, 9. Obtenido de https:// www.revistaespacios.com/a18v39n17/a18v39n17p09.pdf 
Garcia , M. (2003). Gobierno Corporativo y las decisiones de crecimiento empresarial: evidencia en las cajas de ahorro españolas. Islas Canarias: Universidad de Las Palmas de Gran Canaria.

Gomez, G. (28 de 03 de 2020). Universidad de la Sabana. Obtenido de http://www.fccig.com/ pdf_articulos/GobiernoCorporativoylaLeySarbanesJUNIO2005DEFINITIVA.doc2006.pdf. $28 / 03 / 2020$

Hierro, L., \& Herrera, J. (2019). Mecanismo de Intervención del Sector Público: La Empresas Públicas. Recuperado el 22 de 02 de 2020, de Universidad de Sevilla: https:// personal.us.es/Ihierro/Universidad/Materiales_docentes_files/MECANISMOS\%20 DE\%2OINTERVENCIÓN\%2ODEL\%20SECTOR\%2OPÚBLICO.\%20LAS\%20EMPRESAS\%20 PÚBLICAS\%20.pdf. 22/02/2020

Kanov , A. (Mayo de 2003). La propiedad estatal y el desarrollo económico: Presente y futuro. Revista Aportes, VIII(23), 89-100.

Morejon, S., \& Maryury, E. (2016). La teoría organizacional: análisis de su enfoque en una administración pública y su diferencia con la administración privada. Revista Enfoques: Ciencia Politica y Administracion Publica, XIV(25), 127-143.

Nabil, M., \& Oneto, A. (2015). El Gobierno Corporativo en Iberoamérica. El Buen Gobierno de las Empresas Pública. (CAF, Editor) Obtenido de https://www.iimv.org/iimv-wp-1-0/ resources/uploads/2015/04/gobierno-corporativo_web.pdf

OCDE. (2011). Directrices sobre el Gobierno Corporativo de las Empresas Públicas.

Rodriguez, J. (2014). Gobierno corporativo, cultura empresarial en favor de la creación de valor y prevención de conflictos. 14(2), 169-193.

Rojas, L. (2015). Gobierno Corporativo en las Empresas de Propiedad del Estado. Bogota: CESA.

Sanclemente , J. (2019). El Estado accionista: Gobierno de las empresas públicas en Colombia y Francia. Derecho del Estado(42), 249-278.

Sanclemente, J. (Enero de 2019). El Estado accionista: Gobierno de las empresas públicas en Colombia y Francia. Revista Derecho del Estado(42), 249-278.

Vives, X. (2007). El Buen Gobierno de las Empresas Públicas. Occasional Paper OP(7/12). 